\title{
The Pentax airway scope versus the Macintosh laryngoscope: Comparison of hemodynamic responses and concentrations of plasma norepinephrine to tracheal intubation
}

\author{
Heeseung Lee \\ Department of Anesthesiology and Pain Medicine, School of Medicine, Ewha Womans University, Seoul, Korea
}

Background: The Pentax Airway Scope (AWS) is a video laryngoscope designed to facilitate tracheal intubation with a high-resolution image. The Pentax AWS has been reported to cause less hemodynamic stress than the Macintosh laryngoscope. The aims of this study are to investigate the differences in hemodynamic responses and norepinephrine concentrations to tracheal intubation between procedures using he Pentax AWS and the Macintosh laryngoscope.

Methods: Forty patients (American Society of Anesthesiologists class I-II, age range: 18-60 years) were randomly assigned to be intubated with either the Pentax AWS or the Macintosh laryngoscope while under general anesthesia. Routine monitoring, including invasive arterial blood pressure and bispectral index, were applied. Thiopental (4 $\mathrm{mg} / \mathrm{kg})$, fentanyl $(1 \mu \mathrm{g} / \mathrm{kg})$, midazolam $(0.05 \mathrm{mg} / \mathrm{kg})$, and rocuronium $(0.6 \mathrm{mg} / \mathrm{kg})$ were administered for anesthetic induction. Systolic, diastolic, and mean blood pressures and heart rates were recorded pre-intubation, immediately post-intubation (T0), and over the following 10 minutes at one minute intervals (T1, T2, T3, T4, T5...T10). Patient blood was sampled for norepinephrine concentrations pre-intubation (baseline) and post-intubation (T1). Evidence of sore throat was evaluated $30 \mathrm{~min}$ and $24 \mathrm{hr}$ after extubation. Data were transformed to \% basal and expressed as mean \pm SD.

Results: The systolic, diastolic, and mean blood pressure, and heart rate at T0 and T4 were significantly different between the two groups. There was no significant difference in plasma norepinephrine between the two groups. The difference in incidence of sore throat was not significant between the two groups.

Conclusions: Pentax-AWS for tracheal intubation has greater hemodynamic stability than the Macintosh blade laryngoscope. (Korean J Anesthesiol 2013; 64: 315-320)

Key Words: Hemodynamic response, Norepinephrine, Pentax AWS, Sore throat.

\footnotetext{
Received: December 11, 2012. Revised: 1st, December 31, 2012; 2nd, January 21, 2013; 3rd, January 22, 2013. Accepted: January $22,2013$. Corresponding author: Heeseung Lee, M.D., Ph.D., Department of Anesthesiology and Pain Medicine, School of Medicine, Ewha Womans University, 911-1, Mok 5-dong, Yangcheon-gu, Seoul 158-710, Korea . Tel: 82-2-2650-2670, Fax: 82-2-2655-2924, E-mail: leehee@ewha.ac.kr (c) This is an open-access article distributed under the terms of the Creative Commons Attribution Non-Commercial License (http:// creativecommons.org/licenses/by-nc/3.0/), which permits unrestricted non-commercial use, distribution, and reproduction in any medium, provided the original work is properly cited.
} 


\section{Introduction}

The Pentax Airway Scope (AWS) is a video laryngoscope designed to facilitate tracheal intubation using a high-resolution image. The Pentax AWS provides an improved laryngeal view [1] with less movement of the upper cervical spine compared to the Macintosh laryngoscope [2-4], and provides better intubating conditions for novice personnel in pre-hospital settings because the tip of the tube can be monitored continuously during tracheal intubation. Limitations associated with the Pentax AWS include a difficult requirement for adequate mouth opening, high cost and additional maintenance, different skill set requirements, and greater hand-eye coordination requirements [5]. In morbidly obese patients, the Pentax AWS is not recommended as a routine substitute for the Macintosh laryngoscope because the time to intubation takes longer with the Pentax AWS than with the Macintosh laryngoscope [6]. Despite these limitations, the Pentax AWS provides a less stressful environment than the conventional Macintosh laryngoscope during tracheal intubation.

Tracheal intubation causes a reflex increase in sympathetic activity that may result in hemodynamic responses: hypertension, tachycardia, and arrhythmia [7-9]. A change in the plasma concentration of catecholamine also occurs in response to intubation stimulation $[10,11]$. Although transient, the cardiovascular response to tracheal intubation may be harmful to some patients who have a history of myocardial or cerebrovascular diseases. The Pentax AWS is considered less invasive than the Macintosh laryngoscope because it causes minimal displacement of the tongue and other soft tissues of airway structure [12]. Differences in hemodynamic responses to tracheal intubation have been investigated for the Pentax AWS and the Macintosh laryngoscope [13]. Some of these studies failed to demonstrate any differences in hemodynamic responses to tracheal intubation $[14,15]$, while others found significant differences between the two [16]. Currently, differences between the two groups in plasma catecholamine concentrations have not been investigated yet. Therefore, the aim of this study is to investigate differences in hemodynamic responses and norepinephrine concentrations to tracheal intubation between the Pentax AWS and the Macintosh laryngoscope.

\section{Materials and Methods}

After receiving ethical committee approval and written informed consent, 40 patients were enrolled. The patients ranged from 18 to 60 years old and were classified by the American Society of Anesthesiology physical status classification system as I or II. Participants were all supposed to undergo an elective operation that was expected to take one to two hours. Patients were excluded if they had any known cardiovascular diseases, diabetes, endocrine diseases, allergies to any medications, or had anatomical characteristics associated with a difficult airway, such as unstable teeth, a mouth opening of less than $3 \mathrm{~cm}$, or limited neck extension.

Upon entering the operating room, the unpremedicated patients were randomly assigned to the two groups: Group $\mathrm{P}$ received intubation with the Pentax AWS video laryngoscope and Group M received the Macintosh laryngoscope. This study could not be double-blinded to observe hemodynamic changes. Standard monitors (electrocardiogram, pulse oxymeter, noninvasive blood pressure) and invasive radial arterial monitoring were applied. Ice cubes were kept ready for immediate transport and centrifuging of blood in bottles. Patients were placed in a supine position with their necks in a neutral position with a pillow under the occiput. Patients breathed $100 \%$ oxygen for 3 minutes. After ensuring the patients were calm, their baseline blood pressure and heart rate was recorded and the first $4 \mathrm{~mL}$ blood sample (baseline) was taken to measure plasma norepinephrine levels. Induction of anesthesia was performed using thiopental ( $4 \mathrm{mg} / \mathrm{kg})$, fentanyl ( $1 \mu \mathrm{g} / \mathrm{kg})$, and midazolam $(0.05 \mathrm{mg} / \mathrm{kg})$, intravenously. Following loss of consciousness, rocuronium $(0.6 \mathrm{mg} / \mathrm{kg})$ was administered and positive pressure mask ventilation was initiated. After two minutes of positive pressure ventilation, the trachea was intubated with a tracheal tube (standard, curved tube, $7.5 \mathrm{~mm}$ ID for males and $6.5 \mathrm{~mm}$ ID for females) and either the PentaxAWS $^{\circledR}$ video laryngoscope (Pentax Corp., Tokyo, Japan) or the Macintosh laryngoscope (no. 3) by a single anesthesiologist who was an expert in both intubation procedures. Bispectral index (BIS) scores were recorded at the time of tracheal intubation. Anesthesia was then maintained using sevoflurane (end-tidal $2-3$ vol\%) with a $50 \%$ mixture of air and oxygen. The time to intubation was defined as the time taken from when the tip of the blade passes the incisors until the time when the tip of the blade passes out of the incisors after inserting the tracheal tube. If tracheal intubation failed at the first attempt or if a patient's Cormack-Lehane score was greater than three, the patient was immediately excluded from the study. T0 was defined as the time that the tip of the blade passed out of the incisors. T1, T2, T3, T4, T5, and T10 were determined as oneminute intervals from $\mathrm{T} 0$ and hemodynamic responses (systolic blood pressure [SBP], diastolic blood pressure [DBP], mean blood pressure $[\mathrm{MBP}]$, and heart rate $[\mathrm{HR}]$ ) to intubation were recorded at each of these. The second blood samples (T1) were taken to measure plasma norepinephrine values. Sore throat was measured as four grades $30 \mathrm{~min}$ after extubation by nurses in the post-anesthesia recovery unit (PACU) and $24 \mathrm{hr}$ after extubation [17] by anesthetic residents, double-blindly. Sorethroat grades were: none (no sore throat), mild (pain with 
deglutition), moderate (pain present constantly and increasing with deglutition), and severe (pain interfering with eating and requiring analgesic medication).

The blood samples were preserved in ethylenediaminetetraacetic acid (EDTA) tubes, immediately centrifuged $\left(18,000\right.$ rate per $\left.\min , 4^{\circ} \mathrm{C}, 20 \mathrm{~min}\right)$, and the supernatants were meticulously extracted by pipette, divided into aliquots, stored in $\mathrm{a}-70^{\circ} \mathrm{C}$ freezer, and sent to the Neodin Medical Institute (Seoul, Korea) for extraction of norepinephrine. Norepinephrine concentrations were determined by high-performance liquid chromatography (HPLC) with electrochemical detection, using the Catecholamine Plasma Reagent Kit (Bio-Rad, Hercules, CA, USA).

A power analysis performed in a previous article demon-
Table 1. Demographic Data

\begin{tabular}{lcc}
\hline & $\begin{array}{c}\text { Group P } \\
(\mathrm{n}=20)\end{array}$ & $\begin{array}{c}\text { Group M } \\
(\mathrm{n}=20)\end{array}$ \\
\hline Sex $(\mathrm{M} / \mathrm{F})$ & $12 / 8$ & $11 / 9$ \\
Age $(\mathrm{yr})$ & $38.9 \pm 13.3$ & $35.5 \pm 10.5$ \\
Height $(\mathrm{cm})$ & $168.0 \pm 9.3$ & $166.5 \pm 9.8$ \\
Weight $(\mathrm{kg})$ & $64.9 \pm 8.2$ & $66.0 \pm 14.9$ \\
Body mass index $\left(\mathrm{kg} / \mathrm{m}^{2}\right)$ & $23.0 \pm 2.6$ & $23.6 \pm 3.9$ \\
Time to intubation $(\mathrm{sec}) *$ & $18.5 \pm 1.55$ & $12.8 \pm 1.39$ \\
Bispectral index at tracheal intubation & $40.0 \pm 12.9$ & $35.4 \pm 8.7$ \\
Duration of anesthesia (min) & $95.0 \pm 24.2$ & $96.7 \pm 35.9$ \\
\hline
\end{tabular}

Data are expressed as mean \pm SD. Group P: Pentax AWS (Airway Scope), Group M: Macintosh laryngoscope. There was no significant difference between the two groups in demographic data and duration of anesthesia. Time to intubation was significantly longer in Group P than Group $\mathrm{M}(* \mathrm{P}<0.05)$.

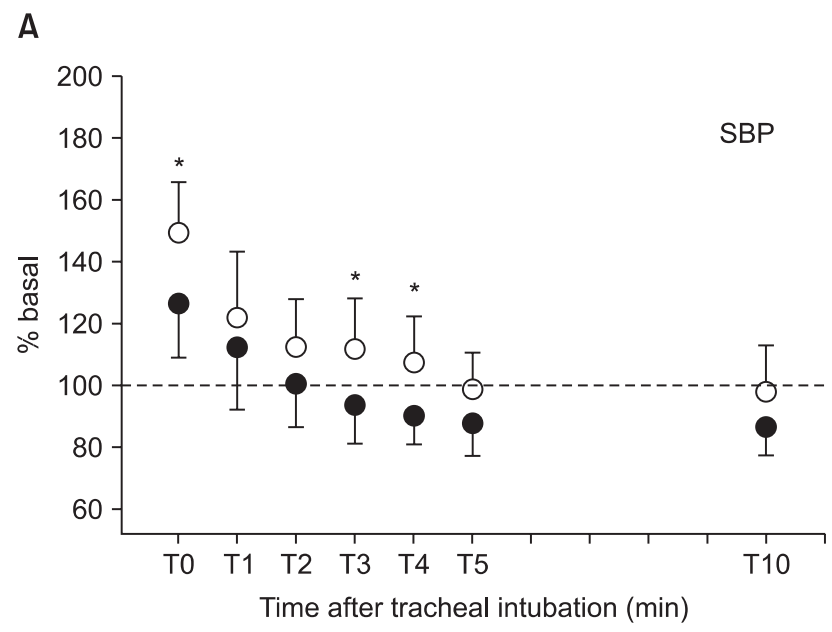

B
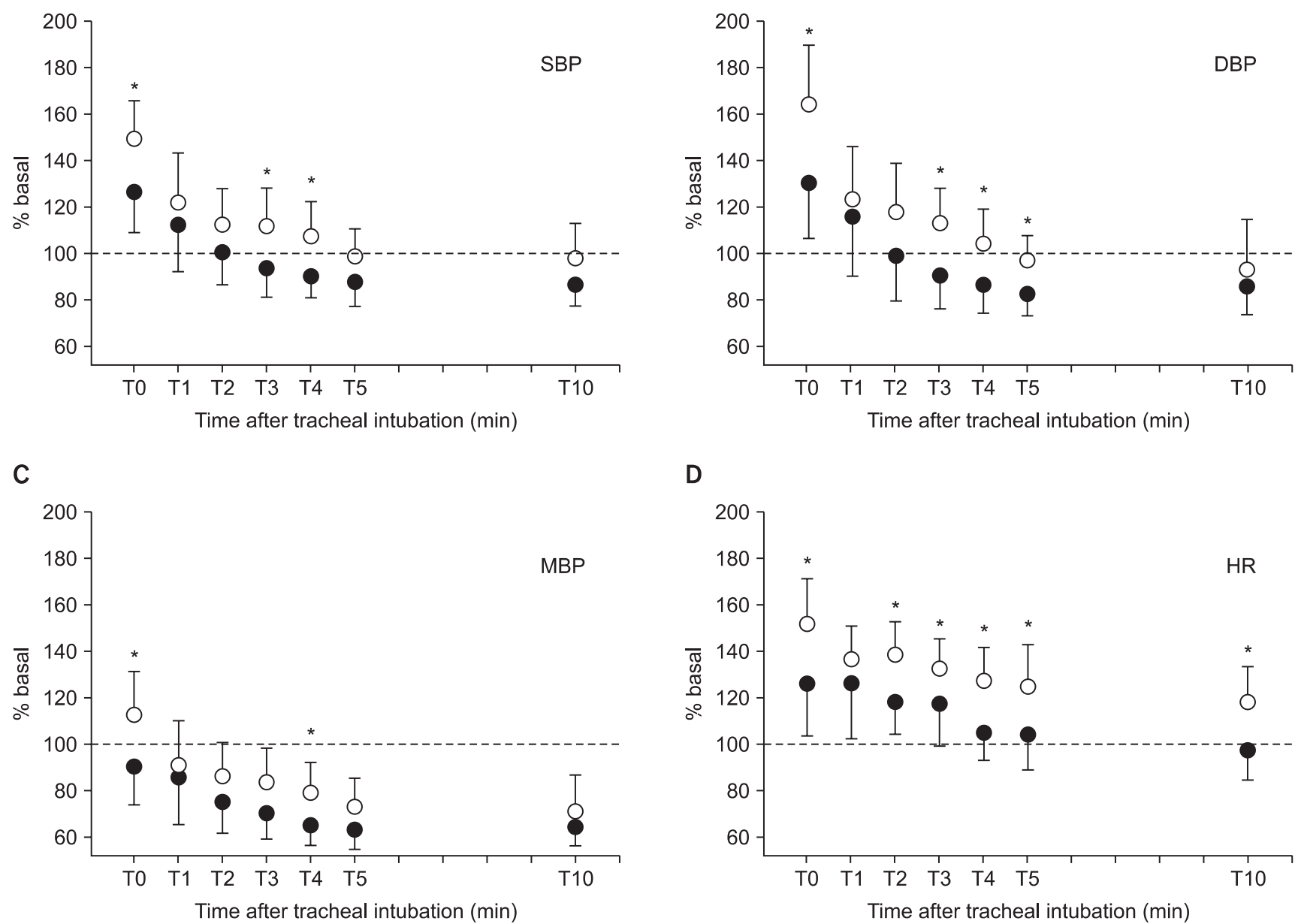

D

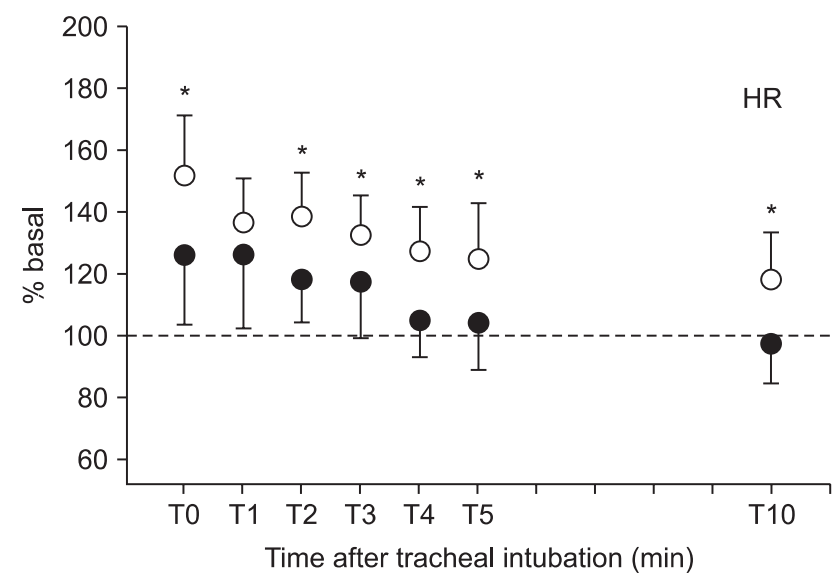

Fig. 1. Hemodynamic responses to tracheal intubation. Data are presented as $\%$ basal: $\%$ basal $=100 \times$ hemodynamic parameters at specific time/baseline hemodynamic parameters. The dotted line of the y intercept at 100 is the baseline value in each panel. Group P $(\mathbf{)})=$ group receiving Pentax AWS (Airway Scope), Group $\mathrm{M}(\bigcirc)$ = group receiving Macintosh laryngoscope, $\mathrm{T} 0=$ time after tracheal intubation 0 minutes, $\mathrm{T} 1-\mathrm{T} 10$ = time after tracheal intubation 1-10 minutes. SBP (A): systolic blood pressure, DBP (B): diastolic blood pressure, MBP (C): mean blood pressure, HR (D): heart rate. Statistical differences in hemodynamic responses to tracheal intubation between Group P and Group M were analyzed by repeated measures analysis of variance (rANOVA), followed by the Sidak multiple comparison test $(* \mathrm{P}<0.05)$. 
strated [18] that a sample size of 20 patients per group was required to achieve a power of $80 \%$ and an $\alpha$ of 0.05 for detection of differences of 20 beats/min or $20 \mathrm{mmHg}$ hemodynamic responses to intubation. All data were expressed as the mean \pm standard deviation (SD). Statistical analyses were performed using GraphPad Prism, version 6.0 (GraphPad Software, Inc., La Jolla, CA, USA). Hemodaynamic responses to intubation were expressed as \% basal: \% basal $=100 \times$ hemodynamic parameters at T0, T1, T2, T3, T4, T5, and T10/baseline hemodynamic parameters. Statistical differences between groups in hemodynamic responses to intubation were analyzed using the repeated measures analysis of variance (rANOVA) followed by the Sidak multiple comparison test. Norepinephrine concentrations were expressed as the $\%$ basal: $\%$ basal $=100$ $\times$ concentration of post-intubation norepinephrine during at $\mathrm{T} 1$ (pg/ml)/concentration of pre-intubation norepinephrine $(\mathrm{pg} / \mathrm{ml})$. Statistical differences between the two groups in norepinephrine concentrations and demographic data were analyzed using the unpaired t-test. Statistical differences of norepinephrine concentration between pre-intubation and post-intubation were analyzed by paired t-test. Statistical differences in the incidence of sore throat at $30 \mathrm{~min}$ and $24 \mathrm{hr}$ after extubation were compared using the Mann-Whitney test. A P value below 0.05 was considered statistically significant.

\section{Results}

Patient demographics, BIS, and duration of anesthesia were similar between Group P and Group M (Table 1). Time to intubation was significantly longer in Group P than Group M (P = $0.010)$.

The \% basal of SBP showed significant differences between groups at T0, T3, and T4 $(\mathrm{P}<0.05$, Fig. $1 \mathrm{~A})$. SBPs immediately after intubation (T0 in Fig. 1A) were $160.5 \pm 22.9 \mathrm{mmHg}$ and $180.0 \pm 26.5 \mathrm{mmHg}$ in Group P and Group M, respectively. The $\%$ basal of DBP showed significant differences between groups at T0 and T3 $-5(\mathrm{P}<0.05$, Fig. 1B). DBPs at T0 (Fig. 1B) were $99.4 \pm 25.9 \mathrm{mmHg}$ and $114.3 \pm 19.3 \mathrm{mmHg}$ in Group $\mathrm{P}$ and $\mathrm{M}$, respectively. The \% basal of MBP showed significant differences between groups at T0 and T4 ( $<<0.05$, Fig. 1C). MBPs at T0 (Fig. 1C) were $115.2 \pm 20.4 \mathrm{mmHg}$ and $135.1 \pm 22.3 \mathrm{mmHg}$ in Group $\mathrm{P}$ and $\mathrm{M}$, respectively. The \% basal of HR showed significant differences between groups at T0 and T2-10 (P< 0.05, Fig. 1D). HRs at T0 (Fig. 1D) were $93.3 \pm 17.5$ beats $/ \mathrm{min}$ and $107.4 \pm 15.9$ beats/min in Group $\mathrm{P}$ and $\mathrm{M}$, respectively. All the hemodynamic responses - SBP, DBP, MBP, and HR-at T0 and T4 demonstrated significant differences between the two groups. The \% basal of DBP at T0 (Fig. 1B) showed the most significant inter-group differences $(\mathrm{P}=0.0018)$.

The $\%$ basal of plasma norepinephrine was not significantly

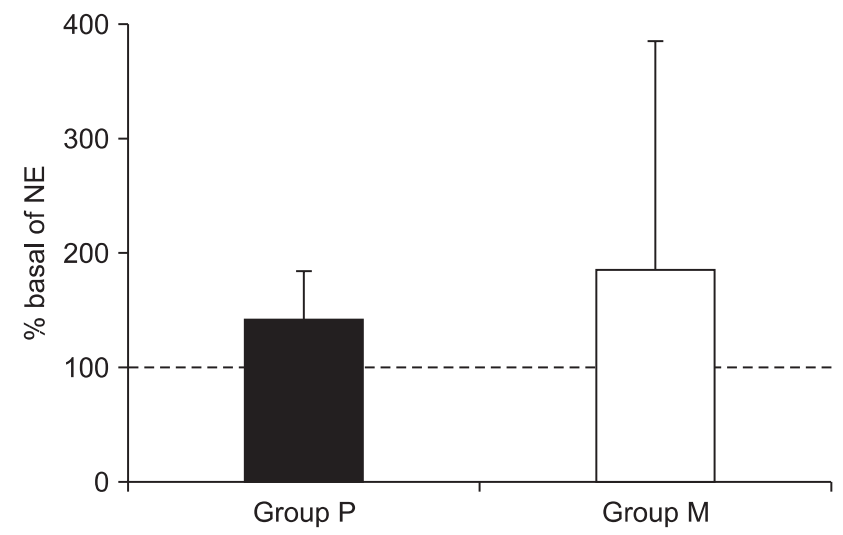

Fig. 2. Concentration of plasma norepinephrine. Data are presented as $\%$ basal: $\%$ basal $=100 \times$ concentration of post-intubation norepinephrine during at $\mathrm{T} 1(\mathrm{pg} / \mathrm{ml}) /$ concentration of preintubation norepinephrine $(\mathrm{pg} / \mathrm{ml})$. The dotted line of the y intercept at 100 is the baseline value. NE=norepinephrine, Group P $(\boldsymbol{\square})=$ group using Pentax AWS (Airway Scope), Group M $(\square)$ = group using Macintosh laryngoscope. Statistical differences in norepinephrine concentration between Group M and Group P were analyzed with an unpaired t-test. There is no significant difference between the two groups in post-intubation concentrations $(\mathrm{P}>0.05)$.

different between Group P and Group M ( $\mathrm{P}=0.211$, Fig. 2). The $\%$ basal of plasma norepinephrine were $145.3 \pm 46.4$ and $204.5 \pm 210.8$ in Group P and Group M, respectively. The actual norepinephrine plasma concentrations were $141.5 \pm 55.6 \mathrm{pg} /$ $\mathrm{ml}$ and $192.0 \pm 91.9 \mathrm{pg} / \mathrm{ml}$ in Group $\mathrm{P}$ and $155.5 \pm 87.4 \mathrm{pg} / \mathrm{ml}$ and $218.8 \pm 86.9 \mathrm{pg} / \mathrm{ml}$ in Group $\mathrm{M}$ at pre- and post-intubation, respectively. The values of norepinephrine at post-intubation increased significantly compared with those at pre-intubation in both groups $(\mathrm{P}<0.05)$.

Mild to moderate sore throat was observed in both Group P (4 to 1 out of 20) and Group M (5 to 1 out of 20) at $30 \mathrm{~min}$ after extubation, although there was no significant difference between two groups. There was no sore throat observed at $24 \mathrm{hr}$ after extubation in either group.

\section{Discussion}

Laryngoscopy and tracheal intubation are well known to be the main causative factors for hemodynamic changes during the induction of general anesthesia. When using the Macintosh laryngoscope, the tips of the laryngoscopic blade are positioned at the vallecula; laryngeal soft tissues and smooth muscles are stretched and pressed upward in the process. Moreover, during Macintosh laryngoscope, the patient's neck is usually extended by the head-tilt and chin-lift maneuver required for straight alignment of the oral, pharyngeal, and laryngeal axis, requiring more force to be applied to the cervical structures [19]. Those factors may contribute to an increase in physiological 
sympathetic activities and elevation of plasma catecholamine concentrations during induction of general anesthesia. When using Pentax AWS, the tips of the blade directly elevate the epiglottis, similar to the tips of the Bullard laryngoscope, and anesthesiologists can expose the glottic opening more efficiently. Thus, less force and physical stress during the tracheal intubation process can be applied to the overall airway structures. Therefore, we tried to investigate the differences in hemodynamic responses to tracheal intubation during induction of general anesthesia between the group receiving either the Macintosh laryngoscope or the Pentax AWS. Also, we tried to evaluate the differences in plasma norepinephrine concentrations between the two groups.

Some researchers failed to show any differences in hemodynamic responses to tracheal intubation between participants receiving intubation with the Macintosh laryngoscope versus the Pentax AWS $[14,15]$, but others were successful in their efforts [16]. In our study, SBP, DBP, MBP, and HR at T0 and T4 were all significantly lower in Group $\mathrm{P}$ than in Group $\mathrm{M}$ - even the time to intubation was significantly longer in Group $P$ than Group M. Continuous arterial BP monitoring and tracheal intubation by a single expert anesthesiologist in both procedures could contribute to better measures of consistent parameters in our patients. Group $\mathrm{P}$ was found to require a similar amount of time to pass through the vocal cords as Group M (data were not shown). The only factor in which Group P took a longer time than Group M, was in the time it took to separate the tracheal tube from the stalk of the Pentax AWS.

Norepinephrine plasma concentrations at the time of tracheal intubation were not significantly different between Group P and Group M. Norepinephrine plasma concentrations at postintubation (T1) were significantly higher than those at preintubation. The time between the first and the second sample in our study was one minute after intubation because it has been reported that mean norepinephrine levels peak one minute post-intubation and then decrease back to baseline [20]. It was more difficult to observe differences in plasma catecholamine concentrations between pre-intubation and post-intubation [21-23]. Only one previous report has shown an elevation in plasma catecholamine concentrations after tracheal intubation [24]. In our results, plasma norepinephrine values were significantly elevated post-intubation (T1) compared to those at pre-intubation in both groups, but there was no significant difference between Group P and Group M.

Although we failed to examine inter-group differences to clarify the meaning of correlations between plasma norepinephrine concentrations and hemodynamic responses to intubation, we did observe intra-group differences between preintubation and post-intubation in all aspects of hemodynamic responses to intubation and concentrations of plasma norepin- ephrine. All hemodynamic responses to intubation, especially DBP at T0, were significantly lower in Group P than Group M. Although the maximum differences in hemodynamic parameters at T0 are only $20-35 \%$ basal between the two groups, these results suggest that patients who are particularly vulnerable to hemodynamic stress during tracheal intubation because of known hypertension, history of coronary artery diseases, and cerebrovascular diseases, including aneurysm, would benefit from receiving the Pentax AWS during tracheal intubation.

Taking all these results into account, our study shows that the Pentax AWS for tracheal intubation under general anesthesia can attenuate hemodynamic stress. Although there was no significant correlation between norepinephrine plasma concentrations and hemodynamic responses to tracheal intubation, the Pentax AWS seems to be a good alternative for safe and smooth induction of general anesthesia, especially for patients susceptible to hemodynamic stress.

\section{References}

1. Suzuki A, Toyama Y, Katsumi N, Kunisawa R, Sasaki S, Hirota K, et al. The Pentax-AWS((R)) rigid indirect video laryngoscope: clinical assessment of performance in 320 cases. Anaesthesia 2008; 63: 6417.

2. Enomoto Y, Asai T, Arai T, Kamishima K, Okuda Y. Pentax-AWS, a new videolaryngoscope, is more effective than the Macintosh laryngoscope for tracheal intubation in patients with restricted neck movements: a randomized comparative study. Br J Anaesth 2008; 100: 544-8.

3. Malik MA, Maharaj CH, Harte BH, Laffey JC. Comparison of Macintosh, Truview EV02, Glidescope, and Airwayscope laryngoscope use in patients with cervical spine immobilization. Br J Anaesth 2008; 101: 723-30.

4. Malik MA, Subramaniam R, Churasia S, Maharaj CH, Harte BH, Laffey JG. Tracheal intubation in patients with cervical spine immobilization: a comparison of the Airwayscope, LMA CTrach, and the Macintosh laryngoscopes. Br J Anaesth 2009; 102: 654-61.

5. Anjum-Nusrath A. Focus on: ophthalmic anaesthesia. Videolaryngoscopy. Current Anaesth Crit Care 2010; 21: 199-205.

6. Abdallah R, Galway U, You J, Kurz A, Sessler D, Doyle DJ, et al. A randomized comparison between the Pentax AWS video laryngoscope and the Macintosh laryngoscope in morbidly obese patients. Anesth Analg 2011; 113: 1082-7.

7. Smith JE. Heart rate and arterial pressure changes during fiberoptic tracheal intubation under genereal anaesthesia. Anaesthesia 1988; 43: 629-32.

8. Finfer SR, MacKenzie SI, Saddler JM, Watkins TG. Cardiovascular responses to tracheal intubation: a comparison of direct laryngoscopy and fiberoptic intubation. Anaesth Intensive Care 1989; 17: 44-8.

9. Smith JE, Mackenzie AA, Sanghera SS, Scott-Knight VC. Cardiovascular effects of fiberscope-guided nasotracheal intubation. Anaesthesia 1989; 44: 907-10. 
10. Boralessa H, Senior DF, Whitwam JG. Cardiovascular response to intubation. A comparative study of thiopentone and midazolam. Anaesthesia 1983; 38: 623-7.

11. Derbyshire DR, Chmielewski A, Fell D, Vater M, Achola K, Smith G. Plasma catecholamine response to tracheal intubation. Br J Anaesth 1983; 55: 855-60.

12. Koyama J, Aoyama T, Kusano Y, Seguchi T, Kawagishi K, Iwashita T, et al. Description and first clinical application of AirWay Scope for tracheal intubation. J Neurosurg Anesthesiol 2006; 18: 247-50.

13. Su YC, Chen CC, Lee YK, Lee JY, Lin KJ. Comparison of video laryngoscopes with direct laryngoscopy for tracheal intubation: a meta-analysis of randomized trials. Eur J Anaesthesiol 2011; 28: 788-95.

14. Turkstra TP, Pelz DM, Jones PM. Cervical spine motion: a fluoroscopic comparison of the AirTraq Laryngoscope versus the Macintosh laryngoscope. Anesthesiology 2009; 111: 97-101.

15. Araki K, Nomura R, Tsuchiya N, Yoshikawa Y. Cardiovascular responses to endotracheal intubation with the Bullard and Macintosh laryngoscopes. Can J Anaesth 2002; 49: 526.

16. Nishikawa K, Matsuoka H, Saito S. Tracheal intubation with the PENTAX-AWS (airway scope) reduces changes of hemodynamic responses and bispectral index scores compared with the Macintosh laryngoscopy. J Neurosurg Anesthesiol 2009; 21: 292-6.

17. Capan LM, Bruce DL, Patel KP, Turndorf H. Succinylcholine- induced postoperative sore throat. Anesthesiology 1983; 59: 202-6.

18. Hirabayashi Y, Hiruta M, Kawakami T, Inoue S, Fukuda H, Saitoh $\mathrm{K}$, et al. Effects of lightwand (Trachlight) compared with direct laryngoscopy on circulatory responses to tracheal intubation. Br J Anaesth 1998; 81: 253-5.

19. Suzuki A, Toyoama Y, Katsumi N, Kunisawa T, Henderson JJ, Iwasaki H. Cardiovascular responses to tracheal intubation with the Airway Scope (Pentax-AWS). J Anesth 2008; 22: 100-1.

20. Koveleskie JR, Safford NC, Mok MS, Steen SN. Effect of $5.0 \mathrm{mg} / \mathrm{kg}$ intravenous lidocaine prior to intubation on the catecholamine and cardiovascular responses. J Anaesth Clin Pharmacol 1996; 12: 175-9.

21. Pernerstorfer T, Krafft P, Fitzgerald RD, Krenn CG, Chiari A, Wagner $\mathrm{O}$, et al. Stress response to tracheal intubation: direct laryngoscopy compared with blind oral intubation. Anaesthesia 1995; 50: 17-22.

22. McCoy EP, Mirakhur RK, McCloskey BV. A comparison of the stress response to laryngoscopy. The Macintosh versus the McCoy blade. Anaesthesia 1995; 50: 943-6.

23. Barak M, Ziser A, Greenberg A, Lischinsky S, Rosenberg B. Hemodynamic and catecholamine responses to tracheal intubation: direct laryngoscopy compared with fiberoptic intubation. J Clin Anesth 2003; 15: 132-6.

24. Fujii U, Tanaka H, Toyooka H. Circulatory responses to laryngeal mask airway insertion or tracheal intubation in normotensive and hypertensive patients. Can J Anaesth 1995; 42: 32-6. 